\title{
Atonía uterina e histerectomía de emergencia en el postparto tardío. Presentación de un caso
}

\author{
Carios Alberto Gómez Fajardo*
}

\begin{abstract}
RESUMEN: Se presenta el caso de una paciente multípara de 34 años de edad quien ingresó al servicio de urgencias obstétricas con un cuadro clínico de shock hipovolémico grave secundario a una atonía uterina en el décimo primer día del puerperio. Se practicaron maniobras de revisión y masaje uterino, y aplicación parenteral de oxitócicos y methergina, sin lograr obtenerse respuesta de la contracción uterina. La gravedad de la situación hemodinámica determinó la necesidad de practicar una histerectomía de urgencia. En este caso se destaca lo tardío de la presentación de la atonía postparto, complicación clásicamente considerada dentro de las primeras horas del período puerperal. Es importante además la posibilidad de una relación etiológica entre lo acontecido a esta paciente con la presentación de una circunstancia generadora de un gran estrés emocional -la muerte accidental del esposo- posiblemente con mediación de mecanismos neuroendocrinos que expliquen la relajación uterina súbita.
\end{abstract}

PALABRAS CLAVES: Atonía uterina, hemorragia obstétrica, histerectomía postparto.

SUMMARY: It is presented a case of severe hemorrhagic shock of a multiparous 34 years old woman in her eleventh postpartum day. It was necessary to perform a emergency hysterectomy because there were not a response to the drugs used to achieve a uterine contraction, and the uterine bimanual massage was fruitless. It is contemplated the possibility of a connection between the emotive stress the patient suffered because of the accidental death of her housband and the beginning of the uterine atony. It is discused a possible physiologyca! explanation of this coincidence, by a neuro-endocrine mechanisms related with the uterine relaxation and the simpathetic stimulation response of a very stressing circumstance.

KEY WORDS: Obstetric Hemorrhage, histerectomy, uterine atony.

\section{Caso clínico}

El día 3 de marzo de 1997 ingresa al Servicio de Urgencias Obstétricas de la Clínica León XIII (ISS Medellín) una paciente de 34 años de edad, con un cuadro de hemorragia de origen uterino y severo shock hipovolémico iniciado de manera súbita, durante su décimo primer día del puerperio.

\section{Antecedentes obstétricos: G4 P4 A0}

Fecha del último parto: 20 de febrero de 1997. Se trató de un parto vaginal normal. Luego del uso de oxitocina para refuerzo de la actividad uterina tuvo una niña sana, de 3.030 gramos. Al día siguiente del parto, 21 de febrero, fue dada de alta, lactando normalmente y con el aspecto de los loquios normales.

El control prenatal de éste último embarazo transcurrió dentro de la normalidad, con controles de hemoglobina de $14 \mathrm{gs}$. y hematocrito de $42 \%$, grupo sanguíneo O positivo. Sus partos anteriores habían transcurrido de igual modo sin complicaciones.

Antecedentes patológicos personales y familiares: negativos Se destaca en los datos proporcionados por la

Médico Ginecólogo y Obstetra. Clínica León XIII. ISS Medellín. paciente luego de su recuperación que el sangrado vaginal masivo se había iniciado dos días después de la muerte accidental de su esposo ( 28 de febrero), un empleado del sector de la construcción.

Su cuadro clínico en el momento del ingreso al servicio de urgencias a los 11 días del puerperio, el 3 de marzo, es el de una severa hemorragia de origen uterino. Tiene una profusa hemorragia vaginal, con hipotensión persistente, palidez, compromiso del estado general y el útero totalmente flácido. Se practica un examen pélvico bajo anestesia y se verifica que no existen desgarros vaginales o cervicales como fuente del sangrado. Se hace masaje uterino y compresión bimanual, y además se aplican parenteralmente methergina y oxitocina, sin lograrse un grado de contracción uterina efectivo.

Ante la gravedad de la situación y la falta de respuesta a las medidas anteriormente relatadas, se determina practicar una laparotomía urgente a través de una incisión mediana infraumbilical. Se encuentra el útero en estado de total relajación muscular, pálido, con aproximadamente $25 \mathrm{cms}$ de longitud. No hay infiltración de sangre en sus paredes, ni hemoperitoneo, no hay otras fuentes de sangrado activo. Se realiza tracción y reparación de redondos, disección del peritoneo vésico-uterino para desplazar anteriormente la vejiga y se practica una histerectomía abdominal con anexectomía izquierda, utilizando Vicril O para la ligadura de los pedículos y 
vasos sanguíneos. Luego se revisa cuidadosamente la hemostasia y se realiza el cierre de la pared abdominal por planos.

Durante el período postoperatorio inmediato la paciente continuó en condiciones críticas, con taquicardia y prolongada hipotensión. Requirió soporte con líquidos parenterales, soluciones coloidales sustitutos del plasma (haemaccel) y transfusiones sanguíneas. Tuvo un episodio de edema pulmonar del cual se recuperó satisfactoriamente con el tratamiento médico. Además, recibió enoxaparina (Clexane), y soporte inotrópico con Dopamina. Se le aplicó Ceftriaxona (Rocefin) en el período perioperatorio. Durante estos episodios la enferma mantuvo una buena función renal, con diuresis normal.

El día 4 de marzo su nivel de hemoglobina era de 5.1 gs. y el hematocrito de $14 \%$. Los niveles de plaquetas y pruebas de coagulación se mantuvieron dentro de los límites normales.

La paciente egresó una semana después de la histerectomía y asistió a una revisión en consulta externa tres semanas más tarde. Allí se encontró asintomática, con buena cicatrización de la herida quirúrgica y en buenas condiciones generales de salud, por lo cual fue dada de alta del servicio de Obstetricia.

Informe de Anatomía Patológica: Utero y anexos izquierdos: Hipertrofia gravídica. Restos placentarios. Historia de Atonía uterina. Trompa y ovario normales.

\section{Discusión}

La hemorragia, junto con los trastornos hipertensivos del embarazo y la sepsis se encuentra entre las causas más importantes de mortalidad materna en el mundo. A pesar de que existen grandes diferencias cuantitativas en las tasas de mortalidad materna (6.6 por 100.000 nacidos vivos en Suecia en 1971 en comparación con más de 200 por 100.000 nacidos vivos en Bangladesh en 1980), estas tres grandes causas de muerte materna se continúan registrando en todos los lugares.

Se ha definido clásicamente la Atonía uterina como la falta de presentación de una contracción uterina eficaz durante el postparto. Generalmente se presenta durante el período de las primeras 24 horas postparto. Se ha llamado "tardía" a la hemorragia importante que se da en un período de tiempo posterior a ese primer día del puerperio.

El presente caso tiene la condición especial de haberse presentado en el décimo primer día postparto, constituye una característica que lo hace singular el lapso de tiempo transcurrido entre el momento del parto y la presentación de la grave complicación.

Khong y Khong con estudios citopatológicos han llegado a la conclusión de que la mayor parte de esta hemorragias postparto tardías (entre 24 horas y 6 meses postparto) se deben a subinvolución del lecho placentario. Esos autores creen que la subinvolución uterina estaría asociada a trastornos en la interacción entre el trofoblasto y el lecho placentario materno. Los hallazgos del presente caso coinciden con esa observación, ya que histológicamente se puso en evidencia la presencia de restos placentarios en un útero subinvolucionado.
Además de la atonía uterina, existe un número importante de causas de hemorragia en el período puerperal, razón por la cual el examen clínico y las ayudas de laboratorio deben orientarse rápidamente a la consideración y evaluación de las otras causas de sangrado. (Tablas 1 y 2). Existe además una importante morbilidad asociada a la necesidad de practicar una histerectomía de emergencia por una hemorragia de origen obstétrico. (Tabla 3).

Tabla 1

\section{CAUSAS DE HEMORRAGIA POSTPARTO}

\author{
Placenta previa \\ Abruptio placentae \\ Placenta accreta \\ CID y otros defectos de la coagulación \\ Atonía uterina \\ Ruptura uterina \\ Laceraciones del cérvix y la vagina \\ Retención placentaria \\ Inversión uterina
}

Tabla 2

\section{FACTORES DE RIESGO ASOCIADOS A ATONIA}
Alta paridad
Parto prolongado o muy rápido
Nivel profundo de anestesia inhalatoria
Corioamnionitis
Hemorragia postparto previa
Embarazo múltiple
Macrosomía hidramnios
Terapia tocolítica
Uso de oxitocina

Tabla 3

MORBILIDAD EN HISTERECTOMIA POR HEMORRAGIA OBSTETRICA (Clark et al)

Transfusión sanguínea
Fiebre
Infección de herida
Coagulopatía dilucional
Trauma de uréter
Paro cardíaco
Tromboflebitis pélvica séptica
Muerte materna

$96 \%$
$50 \%$
$12 \%$
$6 \%$
$4 \%$
$4 \%$
$3 \%$
$1 \%$

El cuadro clínico en el shock hemorrágico grave se caracteriza por un compromiso del estado general de la paciente, con el nivel de conciencia afectado, confusión, desorientación o coma, y las extremidades frías y pálidas. Hay cianosis distal y severa hipotensión (PA Sistólica menor de $80 \mathrm{~mm} \mathrm{Hg}$ ), con taquipnea o disnea. El volumen urinario se puede reducir a menos de $30 \mathrm{ml} /$ hora hasta llegar a la anuria.

En el embarazo normal, cerca del término, están actuando los mecanismos de preparación fisiológica para la pérdida sanguínea normal durante el parto. Esta ha 
sido estimada en $500 \mathrm{ml}$. en el parto vaginal y $1.000 \mathrm{ml}$ en el parto por cesárea. En la gestación normalmente se alcanza un estado de hipervolemia, con aumento del gasto cardíaco del 30 al $40 \%$ al llegar al término. Hay un aumento de 15 latidos por minuto en la frecuencia cardíaca materna con un aumento del volumen sistólico, acompañado de la disminución fisiológica de la resistencia periférica. Los anteriores cambios normales del embarazo determinan el mantenimiento de las cifras de presión arterial y sus particulares cambios a lo largo de la gestación.

En el presente caso se dan las características clínicas descritas en la literatura en relación al shock hipovolémico severo por pérdida masiva de sangre debida a atonía uterina, circunstancia frecuentemente observada en los servicios de urgencias obstétricas. No obstante, llama la atención la presentación tan tardía del evento hemorrágico y la coincidencia de esta complicación del puerperio con el hecho fortuito de la muerte accidental del esposo de la enferma.

Podría considerarse la posibilidad de que este fenómeno se explique por la mediación de mecanismos neuroendocrinos mediados por la liberación masiva de catecolaminas de origen suprarrenal -característica fisiológica de las reacciones emocionales intensas- hasta un determinado umbral de resistencia que desbordó los mecanismos normales de la contracción uterina, ocurriendo un efecto similar al obtenido farmacológicamente sobre el útero grávido con la aplicación de sustancias simpático-miméticas, de amplio uso clínico en la práctica obstétrica con acción relajante de la musculatura uterina.

\section{BIBLIOGRAFIA}

1. Emergency Hysterectomy for Obstetric Hemorrhage Clark et al. Obstet. Gynecol. 1984; 64: 376.

2. Acute Hypotension related to Hemorrhage in the Obstetric Patient. Knuppel and Hatangadi. Obstet. Gynecol. Clin. N.A. 1995; 22(1): 111.

3. Pritchard J., MacDonald P., Gant N. Editors. Williams Obstetrics. Seventeenth Ed. Appleton-Century-Crofts. Norwalk, Conn.
4. Delayed Postpartum Hemorrhage: A morphologic study of causes and their relation to other Pregnancy Disorders Khong and Khong Obstet. Gynecol. 1993; 82: 17-22.

5. Hemorrhagic obstetric emergencies. Mayer, D. Seminars in Anesthesia. 1992; 11(1): 32-42. 